\title{
Residual tactile sensitivity with self-directed stimulation in hemianaesthesia
}

\author{
LAWRENCE WEISKRANTZ, DAREN ZHANG
}

From the Department of Experimental Psychology, University of Oxford, Oxford, UK, and Department of Biology, University of Science and Technology of China, Hefei, Anhui, People's Republic of China

SUMMARY A case of hemianaesthesia caused by right hemisphere stroke was found to have good sensitivity and cutaneous localisation when she touched her impaired hand with her good contralateral hand or with a probe held by that hand. The capacity did not depend solely upon movement or positioning of her right arm, and she could also discriminate whether her own left fingers, as opposed to someone else's inter-digitated fingers, were being touched, but only when she did the touching. Thus, the threshold of the impaired hand appeared to be markedly affected by active involvement of the contralateral limb in delivering the stimulus.

We report here evidence of a surprising degree of residual cutaneous sensitivity in a patient clinically rendered severely hemianaesthetic as a result of stroke.

\section{Case report}

A 56 year old woman was admitted to the Radcliffe Infirmary, Oxford, on 1 November 1985 following an inferior myocardial infarct and right cerebrovascular accident. A CT scan revealed an extensive infarct involving the frontal, parietal, and temporal area of the right hemisphere (fig). Her condition was further complicated on 13 November 1985 by a left deep vein thrombosis and a probable extension of her myocardial infarct on 21 November 1985. She was known to have been hypertensive for years and had already had a milder infarct in January 1985, when a scan indicated involvement limited to the right parietal region. She was admitted to the Rivermead Rehabilitation Centre on 6 January 1986. Examination revealed left visual inattention as well as fifth, seventh, and tenth nerve palsies. There was a severe left-sided hemiplegia with no active movement of the upper limb. She was discharged from Rivermead on 21 March 1986, when her general condition had improved, but sensation and proprioception appeared to be totally absent on her affected side except for some response to deep pressure.

Address for reprint requests: Prof $\mathrm{L}$ Weiskrantz, Department of Experimental Psychology, South Parks Road, Oxford OX13UD, UK.

Received 1 July 1986 and in revised form 13 August 1986. Accepted 15 August 1986

\section{Results}

On conventional testing with von Frey hairs or othe? cutaneous stimuli on her left hand she was found te be very insensitive. She never responded to a hair $\overrightarrow{0}$ less than $0.6 \mathrm{~mm}$ diameter (force of $35 \mathrm{~g}$ ) whe? applied by the experimenter. With larger sizes, she very occasionally responded but showed very rapid adaptation and unreliability, as described classically by Head. ${ }^{1}$ She also showed a considerable reluctance to make forced-choice guesses as to which finger had been stimulated with externally applied and unfelt von Frey hairs. On the fingers of her good hand she showed normal sensitivity.

During one of the breaks in routine tactile testing for another purpose, we noticed her rubbing her inert and insensate left hand with the normal right hand. She was asked if she felt anything, and somewhat to our surprise she said she did. She quietly and insistently maintained that she had feeling in her left hand when it was touched by her own right hand. Therefore we set out to investigate this claim. She was tested on five occasions bridging a period of just under 3 months, starting approximately 3 months after her last stroke. A third researcher was always present (Mr Peter Halligan).

With her eyes closed and head turned away (in this and all subsequent tests), the second finger of her right hand was directed by one of us (LW) to one of the fingers of her left hand and moved gently three times over its distal pad. She was asked to indicate 


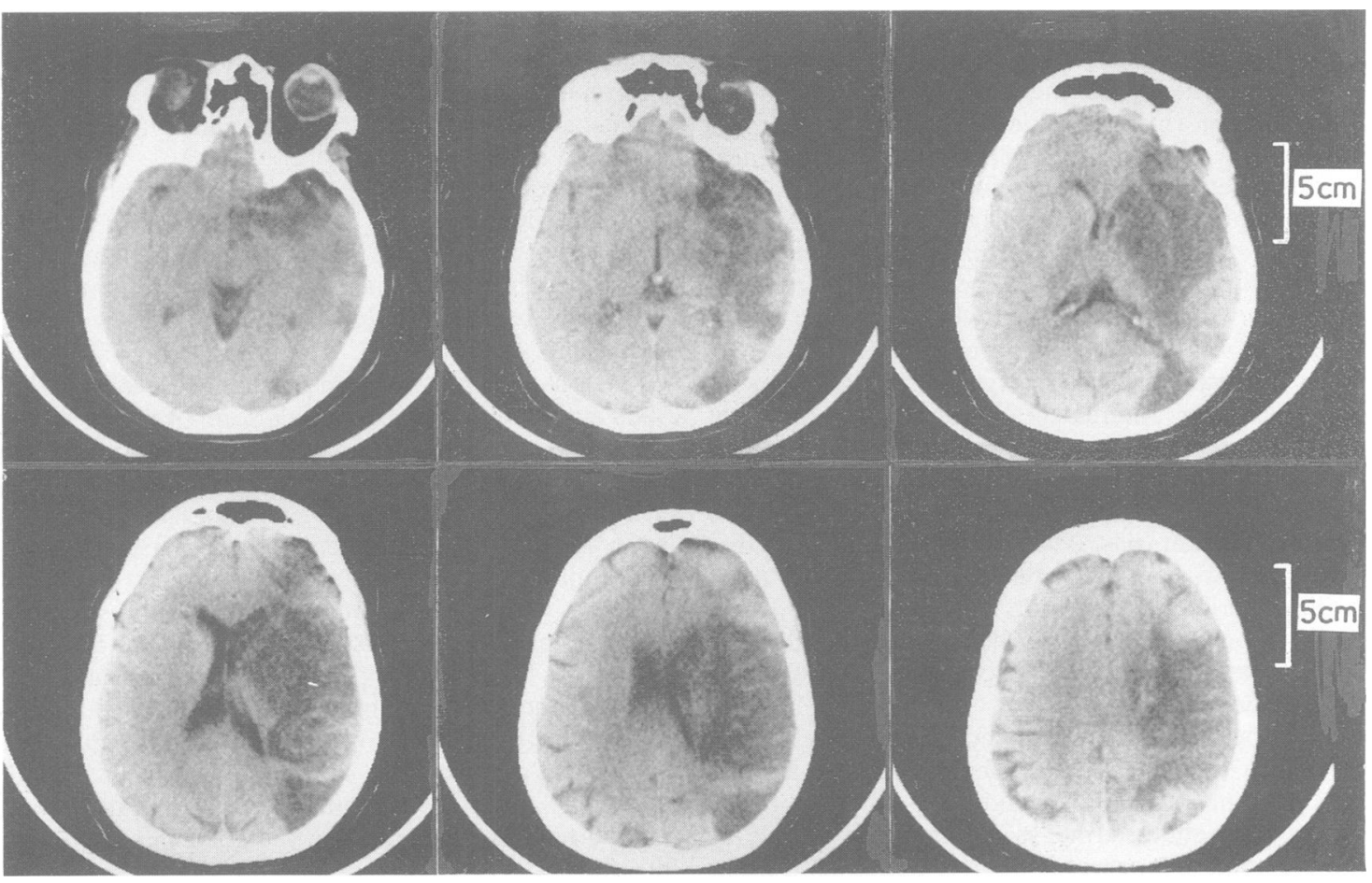

Fig 1 CT scan on 7 November 1985, 14 weeks before initiation of tests

which finger of the left hand was being stimulated, in two blocks of 16 trials each, in a pre-arranged random order. She was extremely accurate. The rank order correlation between finger stimulated and finger identified was $0.839(p<0.0001)$. She remarked that she "felt something definitely every time."

In order to control for proprioceptive and positional cues from her right arm, in all further tests her left hand was positioned so that the finger to be stimulated always fell directly under the stimulating right hand finger. This was done by one of us (DZ) interdigitating his fingers between the fingers of her left hand. Her hand was placed palm upwards upon his inter-digitated hand which, in turn, rested on a soft pillow. A third person elevated her right elbow slightly to ease the movement. Under these conditions, the accuracy over three blocks of 16 trials each was not quite as good, but nevertheless highly significant $(r=0.677, p<0.0001)$. Immediately afterwards an experimenter's finger stimulated her left fingers in the same manner, with her right arm resting, and in 32 trials she replied that she felt nothing at all in 24 trials, and in the other eight her response was non-veridical: she said it felt as though the palm of her hand had been weakly stimulated. In a subsequent test she "felt" 47 out of 48 times when stroked by her own right finger, but on a number of occasions when stimulated in the same manner and approximately the same pressure by the experimenter, felt nothing at all except on a few occasions, when rapid adaptation and non-veridical responding occurred.

Over the 3 months she was tested, her performance gradually deteriorated slightly, but we were able to carry a number of tests, with the following conclusions.

(1) Good performance did not depend solely upon movement or positioning of her right arm such as to approximate contact with her left hand. This was tested by having her "stroke" with her right arm, but with the experimenter's (LWs) finger inter-posed so that it and not her own finger made the actual contact with her left hand. She felt nothing in 16 trials, and remarked that she had "received no information at all."

(2) Good performance did not depend critically upon direct skin-to-skin contact. When she stroked with a blunt pen-top held in her right hand, she could identify the finger of her left hand to which it had been directed $(r=0.733, p<0.0003,32$ trials). Similarly, if she stroked with a von Frey hair (diameter $0.96 \mathrm{~mm}$ ) or a thimble upon the finger of her right 
hand, she reported feeling it on every trial but one (out of 48), but hardly ever if the same was done by the experimenter (four times out of 48).

(3) Good performance did depend upon her left finger being touched. With the experimenter's fingers interdigitated between the fingers of the left hand, the patient's middle finger of the right hand was directed either to one of her own left hand fingers or to the experimenter's fingers in a pre-arranged random order. She correctly identified whether it was one of her fingers or DZs fingers on every trial out of 32 . When this was done by her right hand stroking with von Frey hair (diameter $0.96 \mathrm{~mm}$ ), she correctly assigned it to DZs fingers on every trial (out of 32). When it touched her own fingers, she was correct 15 out of 32. While not perfect, the discrimination is highly significant $\left(\mathrm{p}<0.005, \chi^{2}\right)$. Again, no such discrimination was possible when the action was directed by the experimenter stroking with the von Frey hair. In all but one trial out of 32 she assigned the touch to DZs fingers, although on 16 occasions it had been to her own hand.

(4) The performance was affected by the stimulus applied to the left hand. In the test procedure just mentioned in (3) above, she did not perform at above chance when she used her right middle finger with a thimble upon it. Nor was her performance good when she stroked with the finger nail of her right middle finger (correctly assigned all of the stimuli that touched DZs finger, but only six out of 16 to her own fingers). While this is still highly significant ( $p<$ 0.009, Fisher's exact test), when she stroked with the flat of her right hand finger, she was virtually perfect again (only one error out of 32 ).

\section{Discussion}

Thus, an impressive degree of residual cutaneous sensitivity appeared to be present provided she was actively involved in touching her impaired hand or directing an object to it and an adequate stimulus was used. The sensitivity is highly unlikely to be based upon proprioceptive cues from her right arm. Nor does it derive from the indirect reaction upon her right hand from the pressure placed upon the left hand, because she could discriminate whether the stimulus occurred to her own fingers or the interdigitated fingers that were not her own.

There are some physiological findings consonant with these observations. Sakata et al ${ }^{2}$ described "matching" neurons in area 5 of the unanaesthetised monkey that were driven maximally by bringing two separate parts of the body into contact. Coquery ${ }^{34}$ also described changes in skin sensitivity of the finger $\mathbb{D}$ tip in man caused by finger flexion. While ipsilateralō flexion was inhibitory, it was reported that facilitation? could sometimes be obtained when the flexion 0 occurred contralaterally.

Many questions remain to be explored in this patient (who, however, is not available for testing since her discharge) and others. Is an active command signal necessary to activate the insensitive hand? An:apparatus has been designed to allow a better examination of this question. The threshold of anothero cutaneous phenomenon can be changed markedly by흐 such active self-imposed stimulation. ${ }^{5}$ Another ques- $\frac{\bar{w}^{\circ}}{}$ tion is whether the normal hand must obtain some $\frac{\mathbb{}}{\circ}$ cutaneous feed-back, or whether a command movement itself would suffice. Again, does the phenom- enon depend upon a particular combination of joint $\vec{\circ}$ positioning and contralateral skin contact (as with $\overrightarrow{\vec{\omega}}$ Sakata's "matching" neurons), or would simulta- $\stackrel{\omega}{\circ}$ neous stimulation of skin on both sides of the body be sufficient? Does the cooperation of the contralateralo parts of the body require roughly mirror symmetric ${ }^{\circ}$ regions to be involved? Would the phenomenon also ir be seen on other regions of the body surface? Would $\mathcal{W}^{\circ}$ sensitivity occur if the inert impaired hand were $N$ moved to make contact with the good hand fixed in 을 position?

Nothing can now be said about the frequency of occurrence of such a phenomenon, for which we cin discover no previous clinical reports. But given th $\overrightarrow{8 t} \overrightarrow{0}$ the patient had a common and unremarkable clinicial history of severe hemianaesthesia plus hemiplegia, $\square$ with an extensive unilateral lesion, it seems likely that the phenomenon seen in her may not be unique.

\section{References}

1 Head H. Sensation and the cerebral cortex. Brain 1918;41:57-253.

2 Sakata H, Takaoka Y, Kawarasaki A, Shibutani $\mathrm{H}$ Somatosensory properties of neurons in the superior parietal cortex (area 5) of the rhesus monkey. Brain? Res 1973;64:85-102.

3 Coquery $\mathrm{J}-\mathrm{M}$. Role of active movement in control of afferent input from skin in cat and man. In: Gordon G, ed. Active Touch. Oxford: Pergamon Press, 1978:161-9.

4 Coquery J-M, Coulmance M, Leron M-C. Modifications des potentiels évoqué corticaux somesthesiques duranto des mouvements actifs et passifs chez l'homme. Electroencephalogr Clin Neurophysiol 1972;33:269-76. N

5 Weiskrantz L, Eliot J, Darlington C. Preliminary obser-vations on tickling oneself. Nature 1971;230:598-9. 